\title{
Closed-loop monitoring by anesthesiologists- a comprehensive approach to patient monitoring during anesthesia
}

\author{
Manisha Manohar, Bhavna Gupta, and Lalit Gupta \\ Department of Anesthesia and Critical Care, Maulana Azad Medical College and Lok Nayak Hospital, \\ New Delhi, India
}

The presence of a trained and experienced anesthesiologist is the main determinant of patient safety during anesthesia. Monitoring is defined as observation of a patient by a physician and analysis of the quality of sedation or anesthesia over a time period. Administration of anesthesia requires continual assessment of the physiological state of the patient as well as the adequacy of anesthesia. Although monitoring of the patient using monitors supplements clinical observation, the former is by no means a substitute for the latter. Monitoring and recording may be performed using either automated computerized methods or manual measurement. Automated computerized methods have the advantage of saving the recorded information and observing trends over time. Association of Anaesthetist of Great Britan and Ireland standards of monitoring include the following [1]:

1. The presence of a qualified anesthetist,

2. Monitoring the patient's oxygenation, ventilation, circulation, and temperature

a) Ventilation: Assessed by measuring end-tidal carbon dioxide and inspired anesthetic gases.

b) Oxygenation: Assessed by measuring peripheral oxygen

Corresponding author: Bhavna Gupta, MBBS, DA, DNB.

Department of Anesthesia and Critical Care, Maulana Azad Medical College and Lok Nayak Hospital, Bahadur Shah Zafar Marg, Balmiki Basti, New Delhi 110002, India

Tel: 91-8527686660, Fax: 91-1126853108

Email: bhavna.kakkar@gmail.com

ORCID: https://orcid.org/0000-0002-3108-0408

Received: February 11, 2018.

Revised: February 22, 2018.

Accepted: February 25, 2018.

Korean J Anesthesiol 2018 October 71(5): 417-418

https://doi.org/10.4097/kja.d.18.00033 saturation and inspired anesthetic gases.

c) Temperature: Core temperature monitoring (e.g., tympanic membrane, distal esophagus, nasopharynx, etc.) is used to monitor intraoperative hypothermia, prevent over-heating, and facilitate detection of malignant hyperthermia.

d) Circulation: Assessed by measuring heart rate and non-invasive blood pressure every five minutes, and through electrocardiogram analysis.

Minimum basic standard monitoring during general anesthesia includes pulse oximetry, electrocardiogram, monitoring of non-invasive blood pressure, end-tidal carbon dioxide, and airway pressure. The use of a nerve stimulator is indicated whenever a muscle relaxant is used. During regional anesthesia and sedation procedures, appropriate monitoring should be performed using pulse oximetry, measurement of non-invasive blood pressure, and electrocardiography, at a minimum [1]. In principle, monitoring of physiological variables provides information and feedback on the body's response to therapeutic interventions or changing clinical conditions. This allows fine-tuning of patient management to achieve optimal outcomes and minimization of complications.

Clinical observation and assessment by a vigilant anesthetist are essential for safe patient care during anesthesia. Appropriate clinical observations include the color of the mucosa, sweating, lacrimation, movement of the patient, response to a surgical stimulus, excursions of the chest wall, and movements of reservoir bag/bellows etc. A simple palpation of the radial/carotid pulse can indicate the volume status of the patient. Other clinical monitoring parameters include measurement of urine output, blood loss, and auscultation of heart sounds and breath sounds. Standard monitoring requires that measurements be performed every 5 minutes. Changes in clinical signs often precede abnor-

(c) This is an open-access article distributed under the terms of the Creative Commons Attribution Non-Commercial License (http://creativecommons.org/ licenses/by-nc/4.0/), which permits unrestricted non-commercial use, distribution, and reproduction in any medium, provided the original work is properly cited. 


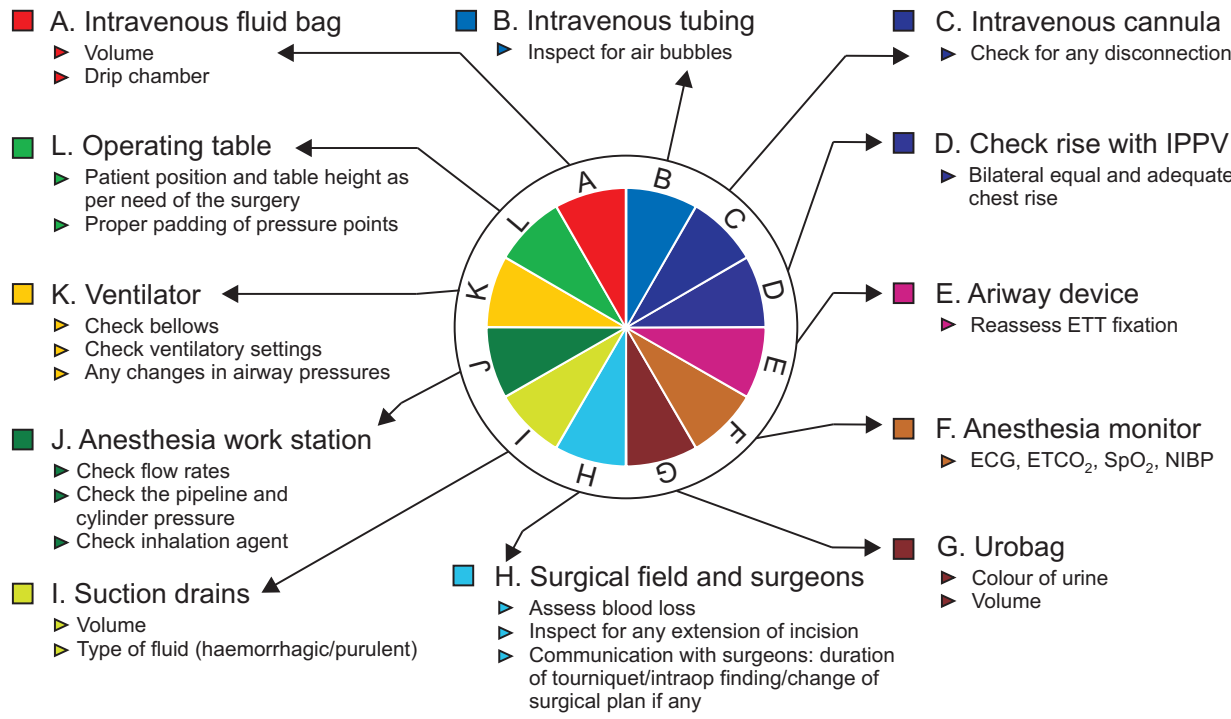

Fig. 1. Example of closed-loop monitoring. IPPV: intermittent positive pressure ventilation, ETT: end tracheal tube, ECG: electrocardiogram, $\mathrm{ETCO}_{2}$ : end tidal $\mathrm{CO}_{2}, \mathrm{SpO}_{2}$ : peripheral oxygen saturation, NIBP: non-invasive blood pressure. malities detected by monitoring equipment. For example, just after induction, or after administering local anesthetic during a subarachnoid block, hypotension is anticipated. While monitors may not pick it up immediately, clinical assessment and palpation of the pulse can detect hypotension earlier. Similarly, if breathing circuit get disconnected, it can be detected by direct observation even before saturation falls on monitor. Most of the alarm signs are seen on the monitor only after an event occurs in the surgical field (e.g., tachycardia on the monitor, after blood loss); this indicates that assessing the surgical field should also be a component of anesthetic monitoring.

Till date, technological advances are unable to alert us about displaced intravenous lines, urinary catheters, breathing circuits, or surgical drains. To ensure comprehensive monitoring of the patient at regular intervals, we suggest a closed-loop monitoring method. The principles of closed-loop monitoring can be used in regional anesthesia, general anesthesia, monitored anesthesia care, and the intensive care unit. Closed-loop monitoring includes closed-loop visual inspection of the patient, anesthesia machine, standard monitors, and surgical field at regular intervals in a predetermined sequence so that none of the parameters are missed. The main sequence is as follows: Patient (clinical assessment, pulse, airway, chest movements, intravenous lines, rate and amount of fluid infusion, urine output, surgical field, blood loss, suction drains, etc.) $\rightarrow$ Monitor $\rightarrow$ Anesthesia workstation (including vaporizers, ventilators, etc.), $\rightarrow$ Operating table and patient record sheet $\rightarrow$ and back to Patient (Fig. 1). Simultaneously, it needs to be ensured that alarm limits are set at an appropriate level so that the changes can be detected early. Collection of all data on a data sheet is not a huge task, but it is an indispensable part of monitoring the patient, as it provides information on the entire duration of the surgery and can also be used later as part of the medical records. The closed-loop monitoring approach will not only prevent adverse incidents or accidents but will also help in improving the safe provision of anesthesia and reducing anesthesia-related morbidity/mortality.

\section{ORCID}

Manisha Manohar, https://orcid.org/0000-0002-9781-2544

Bhavna Gupta, https://orcid.org/0000-0002-3108-0408

Lalit Gupta, https://orcid.org/0000-0001-7291-5961

\section{Reference}

1. Checketts MR, Alladi R, Ferguson K, Gemmell L, Handy JM, Klein AA, et al. Recommendations for standards of monitoring during anaesthesia and recovery 2015: Association of Anaesthetists of Great Britain and Ireland. Anaesthesia 2016; 71: 85-93. 